\title{
Comparative Analysis of Transmission and Switching Technologies
}

\author{
Yanhong Chen ${ }^{1, a}$, Xiaoxia Wang ${ }^{1}$ and Qinghua $\mathrm{Wu}^{1}$ \\ ${ }^{1}$ High-tech Research Institute, No.12 Fangongting South Street, Qingzhou City, Shandong Province, China
}

\begin{abstract}
This paper compares the advantages and disadvantages of OTN, SDH, WDM, ASON and other transmission and switching technologies, as well as the carrying schemes, and focuses on the analysis of the advantages of OTN-based ASON network in networking, capacity expansion, protection and other aspects.
\end{abstract}

In the era of uwb metropolitan area network, optical network is evolving from SDH to OTN in order to meet the requirements of large broadband, IP, high reliability and other ip-centric services.

\section{OTN, SDH AND WDM}

SDH focuses on the electrical layer processing of business, has flexible scheduling, management and protection capabilities, and has perfect OAM functions. However, SDH is unable to meet the needs of rapid business growth because it takes vc- 4 as the basic crossscheduling particle and adopts single-channel line, and capacity growth and scheduling particle are limited. WDM focuses on the optical layer processing of services, and the transmission characteristics of multi-wavelength channels make it have the advantage of large-capacity transmission, but it is still inferior to SDH in scheduling, protection, management and other aspects.

The line characteristic of OTN is that the transmission setting of any digital customer signal is independent of the customer specific characteristics (that is, customer independence). SDH/MSTP has a variety of service transmission functions, rich management and protection functions. WDM improves bandwidth utilization and implements transparent service transmission. OTN inherits the advantages of WDM network capacity and SDH network flexibility and perfectly meets the current business requirements. OTN not only applies SDH's operability and manageability to WDM, but also has the advantages of SDH's flexibility and reliability and WDM's large capacity. Pairs of these techniques are shown in table 1.

OTN effectively inherits the advantages of SDH and WDM, perfectly supports the current business requirements, and gradually realizes the goal of "one network, multiple business hosting". OTN has created a new situation that optical layer is independent of electrical layer. It can complete the functions of signal transmission, multiplexing, routing, switching, monitoring and other functions on optical layer, and guarantee its performance index and survivability. OTN supports various upper layer technologies and is an ideal base transport network to adapt to the evolution of various communication networks.

Table 1. Comparison of OTN, SDH and WDM Carrying schemes

\begin{tabular}{|c|c|c|c|}
\hline \multicolumn{2}{|c|}{ OTN compared with SDH } & \multicolumn{2}{|c|}{$\begin{array}{l}\text { OTN compared with } \\
\text { traditional WDM }\end{array}$} \\
\hline OTN & SDH & OTN & WDM \\
\hline $\begin{array}{c}\text { Broad } \\
\text { bandwidth, } \\
\text { convenient } \\
\text { capacity } \\
\text { expansion }\end{array}$ & $\begin{array}{c}\text { Narrow } \\
\text { bandwidth }\end{array}$ & $\begin{array}{c}\text { Multiple } \\
\text { networking } \\
\text { capabilities }\end{array}$ & $\begin{array}{c}\text { Poor } \\
\text { networking } \\
\text { skills }\end{array}$ \\
\hline $\begin{array}{l}\text { Large particle } \\
\text { scheduling }\end{array}$ & $\begin{array}{c}\text { Pellet } \\
\text { scheduling }\end{array}$ & $\begin{array}{l}\text { Strong and } \\
\text { flexible } \\
\text { scheduling } \\
\text { ability }\end{array}$ & $\begin{array}{l}\text { Business } \\
\text { scheduling is } \\
\text { inflexible }\end{array}$ \\
\hline $\begin{array}{c}\text { High } \\
\text { efficiency of } \\
\text { business } \\
\text { encapsulation } \\
\text { and transparent } \\
\text { business } \\
\text { transmission }\end{array}$ & $\begin{array}{c}\text { low } \\
\text { business } \\
\text { packaging } \\
\text { efficiency }\end{array}$ & $\begin{array}{l}\text { A variety of } \\
\text { sound } \\
\text { protection } \\
\text { mechanisms }\end{array}$ & $\begin{array}{l}\text { The } \\
\text { protection } \\
\text { mechanism } \\
\text { is imperfect }\end{array}$ \\
\hline $\begin{array}{l}\text { Multistage } \\
\text { series } \\
\text { monitoring }\end{array}$ & $\begin{array}{c}\text { Single } \\
\text { stage } \\
\text { monitoring }\end{array}$ & $\begin{array}{c}\text { Rich } \\
\text { overhead } \\
\text { management }\end{array}$ & $\begin{array}{c}\text { Simple OSC, } \\
\text { unable to } \\
\text { accurately } \\
\text { manage } \\
\text { channels }\end{array}$ \\
\hline $\begin{array}{l}\text { Out-of-band } \\
\text { FEC }\end{array}$ & $\begin{array}{c}\text { No FEC or } \\
\text { in-band } \\
\text { FEC }\end{array}$ & & \\
\hline
\end{tabular}

\section{OTN, SDH and ASON}

Both SDH and OTN are typical transmission network

a Corresponding author: 395179801@qq.com 
technology systems. And ASON is has nothing to do with the specific transmission technology of network architecture, its greatest progress is in the original system of transmission network technology increases the control plane, and transferring the ownership of the original management plane function in the control plane, adopts the distributed control mechanism to replace the past a single centralized management mode, introduced the concept of dynamic exchange, thus will exchange, transmission, data real together, the relationship between the three is shown in figure 1.

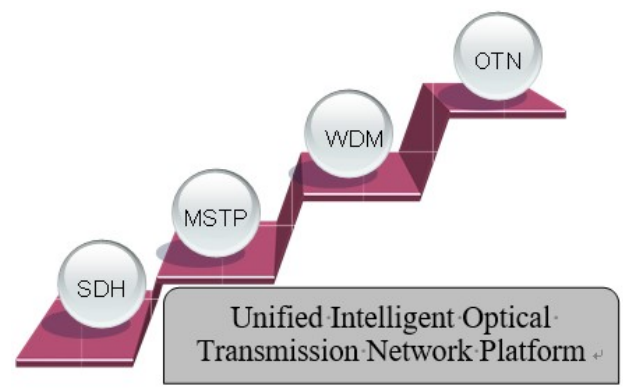

Static network $\square$ Dynamic network

Figure 1. Relationship between SDH, OTN and ASON

After the traditional optical transmission network USES the ASON technology, makes the traditional concept of multi-layer network structure becomes simple and flat, optical network layer to carry business directly, avoiding the traditional Internet business to upgrade when received multiple restrictions, can satisfy the user dynamic allocation of resources, effective protection of resilience and wavelength requirements for the application of new business and so on. The concept and idea of ASON can be extended to different transmission network technologies and has universal adaptability. Therefore, it can be said that the concept of ASON is not only a historical breakthrough in the concept of traditional.

\section{ASON network based on OTN}

With the development of the network, for the large-grain broadband service, the pure information transmission capacity has been unable to measure the efficiency of the transmission network, and the network and service survivability are more dependent on the framework of flexible transmission network to guarantee. Therefore, the concept of network readiness was proposed. That is:

Network readiness $=$ information transmission capability + business control capability + network operation capability

Therefore, the transmission capacity relying on OTN alone cannot meet the requirements of the grid, and an otn-based ASON network is needed, as shown in figure 2. In general, the advantages of otn-based ASON network are reflected in the following six aspects.

\subsection{Networking}

First, it can realize flexible and efficient scheduling and networking of large granule services. Based on ODUk's high-capacity electrical cross matrix, multiple optical line directions are supported to ensure flexible scheduling of large-particle services in the electrical layer. The basic particles of dispatching business are GE and $2.5 \mathrm{~g}$ POS, which can improve the efficiency. Combined with wavelength - based scheduling, efficiency and flexibility can be improved. Second, network survivability has been greatly improved. Traditional WDM has limited protection methods because of its single alarm detection means, no switching protocol is defined and no powerful cross-scheduling module. OTN supports electric cross matrix, can provide special automatic protection switching overhead, support comprehensive alarm detection, with a good foundation to provide a variety of protection methods.

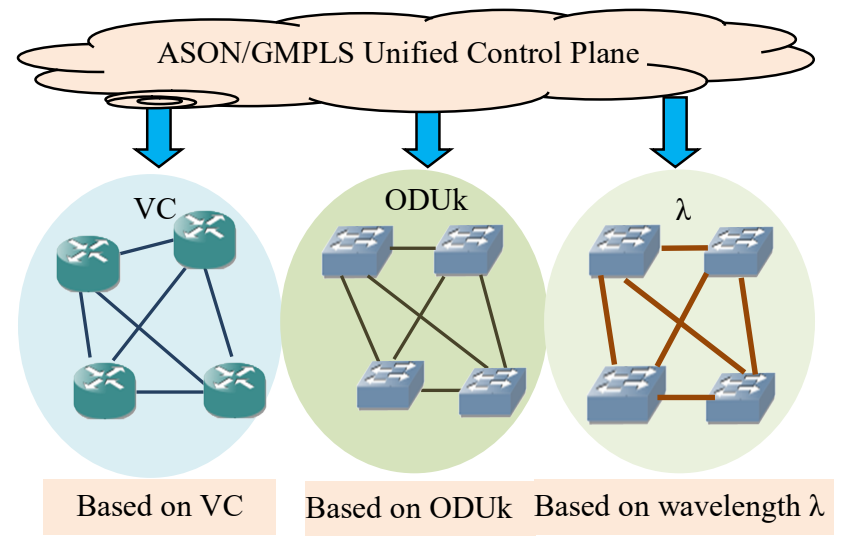

Figure 2. Multi-layer ASON Network Based on OTN

\subsection{Capacity expansion}

Automatic resource discovery for network expansion. The network has capacity expansion needs. When nodes need to be added, the nodes on both sides of the newly added nodes can automatically find new nodes, update the database, and automatically display the new topology on the network management. When the business is damaged, the business automatically finds out that the new route through the new node can realize the non-damage recovery of the business.

\subsection{Clock}

Clock information transparent transmission, can provide multiple SDH subnet mixed transmission. G. 709 provides an asynchronous mapping mode from stm-n to OPUk, in which OPUk signal is generated by the clock of OTN device itself and is independent of stm-n signal, and a positive/negative/zero adjustment mechanism is used to tolerate a certain frequency deviation. In this way, multisubnet mixed transmission can be realized. OTN technology can better adapt to the construction requirements of trunk network at all levels.

\subsection{Protection}

One is to provide wavelength level optical channel 
protection, photonic network connection protection. These protection methods have been widely used in traditional WDM systems. The other is to provide protection of subnet connection based on ODUk. This is a relatively mature protection method of OTN at present, which has little relation with network topology. It provides protection by using the double-generation selective receiving function of electrical layer intersection, which can be applied to ring, chain and Mesh. Third, provide ring network protection based on ODUk. OTN can support the ring network protection of SDH MSP ring, for example, because the electrical crossover ability brings great flexibility, the electric layer alarm carried by OTUk frame is the trigger condition of ring network inversion; Fourth, MESH protection based on ODUk and wavelength. This protection method has the characteristics of ASON. Combined with ROADM's OTN/ASON equipment, it can provide multiple path recovery in both ODUk and wavelength layers, greatly improving the network survivability.

\subsection{Business}

One is the multilayer ASON network, with more transmission options. Otn-based ASON supports ODUk ASON, which enriches the ASON network layer and enables more modes of delivery for demanding customer businesses. For small granular businesses with remote scheduling, end-to-end ASON scheduling and management can be supported by means of SDH network and OTN network docking. The second is to provide various business Level agreements (slas) Level of business. Otn-based ASON provides SLA business of ODUk; ROADM provides wavelength SLA business. Third, to provide full service access to adapt to future network development. OTN defines a variety of mapping methods, so that different types of client side signals have a suitable path to adapt to the OTN network; OTN defines virtual cascaded frames that are used to deliver large granular services over ODUk; G.709 provides encapsulation of package-based user data, that is, Ethernet frames are encapsulated directly through the GFP (Generic Frame Protocol). GFP encapsulation protocol (g.7041) is a general method to encapsulate arbitrary packet signals into fixed rate signals (such as OPUk), which can guarantee the support of new business in the future.

\subsection{Management}

Provides end-to-end channel OAM capability. Traditional WDM devices can only monitor a small amount of optical layer information, such as optical power, and cannot realize the monitoring based on business channel. The operation and maintenance management remains unchanged, and they cannot provide the protection based on business channel and other functions. OTN, because it draws on the advantages of SDH, defines perfect and rich monitoring bytes in the frame structure, which can realize monitoring based on business channels. Serial monitoring bytes carry information of source and host nodes, which makes monitoring function more powerful. The improvement of end-to-end channel OAM function enables OTN network to have the operation, maintenance and management functions similar to SDH network, and to provide protection and other functions based on business channels. The embedded network management channel reduces the management cost. Traditional WDM systems cannot provide embedded network management channels, and its management information needs to be realized through external independent optical modules. OTN provides special general communication channels, which can realize embedded network management. The embedded network management channel adopts the electric monitoring mode, which can avoid the waste of resources in the optical monitoring mode. Each line of business can provide a network management channel to achieve redundant backup; The use of embedded network management does not affect the use of non-embedded network management, traditional monitoring methods can still support.

\section{References}

1. Zeng Shan, Zhang Haibo, Zhang Liangkai. Application of otn transmission network technology in power communication system (Electronic Test, Issue no.12, 2018).

2. Mengqi Chen, Study on the carrying mode of large particle and large connection based on OTN technology (Telecom Engineering Technics and Standardization, Issue no.9, 2018).

3. Ming Gao. Study on ASON programming method based on OTN (Communications Management and Technology, Issue no.1, 2014). 